\title{
Editorial
}

\section{Clinical Trials with Oncolytic Viruses: Current and Future Prospects}

Reviewing the research in the field of oncolytic virus therapy (OVT) of the past two decades, it is inspiring to see the enormous amount of success accomplished by the scholars of this innovative therapeutic technique. Though the experimental trials have been ongoing from 1990s, however, it took a leap forward with approval of the clinical trials in China, 2005. The world's first oncolytic virus to be approved by their government was adenovirus (with E1B 55K gene deletion) for head and neck cancer therapy along with chemotherapy. ${ }^{1}$

The original idea of using viruses as an oncolytic dates back to the early decades of the 20th century, but the concept of using genetically modified viruses is recent. In those times, it was observed that patients with various cancers had undergone spontaneous regression of the tumor mass followed by vaccinations (rabies) or after a viral infection. Series of experimental studies followed based on these observations, confirming the role of viruses (Newcastle disease virus, influenza virus), but due to lack of modern knowledge of molecular biology, virology, genetics and biotechnology, it remained an unexplored arena., ${ }^{2,3}$

Currently, OVT epitomizes a unique class of cancer therapeutics with a distinctive mechanism of action. The viruses selectively replicate and spread within the tumor without affecting the normal noncancerous cells of the host. ${ }^{4,5}$ There are two types of oncolytic viruses: (i) the naturally replicating viruses preferentially in the tumor cells, e.g. Seneca valley virus, myxoma virus and (ii) the genetically modified viruses, e.g. adenovirus, herpes simplex virus. ${ }^{6}$ The simplified mechanism of action of OVT can be explained in two phases. In the first phase, the oncolytic viruses infect, replicate and kill the cancerous cells, eliciting an immunogenic cell death response. Following which in the second phase, the apoptotic tumor cells are engulfed by the antigen presenting cells and the tumor-associated antigens are processed. Resulting effect is a cytotoxic immune response (involving CD4+ and CD8+ T-cells), further helping in the tumor eradication process. ${ }^{1,2,7,8}$

Few advantages of OVT which makes it a high therapeutic index value treatment modality includes (i) tumor selective replication, nonpathogenic, hence minimal systemic side-effects, (ii) in situ viral dose amplification as opposed to other drug pharmacokinetics, (iii) genetically manipulated in-built safety measures and (iv) lastly, less chances of a resistance development. ${ }^{6,7}$ Then again, there is an issue with respect to its mode of delivery. The main methods being employed are intratumoral injection and systemic administration. Intratumoral injection can be advantageous for the easily accessible solid tumors, whereas systemic can prove to be beneficial for the nonsolid tumors and undiagnosed metastatic deposits. ${ }^{9}$

Now coming to its important aspect the clinical trials and outcomes. Numerous trials have been conducted, most of them being in phase I or phase II. In 2014, two OVT phase II trials were completed with an oncolytic vaccinia JX-594 that expresses GM-CSF (Pexa-Vec) in hepatocellular carcinoma patients (sponsored by Jennerex, Inc.) and with an oncolytic reovirus (Reolysin, sponsored by Oncolytic Biotech) in patients with metastatic melanoma. A recent report by Erika et al (2015) states that not less than 50 clinical trials are ongoing with OVT in cancer patients. Most of these trials are exploring the reovirus activity in patients affected by multiple myeloma, head and neck cancers (HNC), breast carcinoma, melanoma, lung cancer, pancreatic carcinoma, prostate cancer, reproductive tract neoplasms or pediatric solid tumors. ${ }^{10}$

With respect to HNC, phase II and III trials are ongoing with reolysin (wild-type reovirus serotype 3 Dearing, developed by Oncolytics Biotech) in combination with carboplatin and paclitaxel. Phase I trial with GL-ONC1 (highly attenuated oncolytic vaccinia virus developed by Genelux) is being tested along with combination with cisplatin and radiotherapy. HF10 (a spontaneous attenuated variant of HSV1) as a single agent has been tested in refractory HNC cases which is also in phase I trial. ${ }^{10}$

To conclude, there has been pioneering research in the field of cancer biology, genetics and immunotherapy facilitating the designing of targeted cancer therapies. Though development of targeted therapies is challenging due to heterogeneity of the cancer, the preliminary results of these trials look promising. Hence, the rational combination of OVT with immunotherapy, chemotherapy or radiotherapy might be most efficient and in near future be the standard clinical protocols for management of cancer. 


\section{REFERENCES}

1. Wong HH, Lemoine NR, Wang Y. Oncolytic viruses for cancer therapy: overcoming the obstacles. Viruses 2010;2(1):78-106.

2. Mullen JT, Tanabe KK. Viral oncolysis. The Oncologist 2002;7(2):106-119.

3. Russell SJ, Peng KW, Bell JC. Oncolytic virotherapy. Nature Biotechnology 2012;30(7):658-670.

4. Atherton MJ, Lichty BD. Evolution of oncolytic viruses: novel strategies for cancer treatment. Immunotherapy 2013;5(11):1191-1206.

5. Peters C, Rabkin SD. Designing herpes viruses as oncolytics. Molecular Therapy—Oncolytics 2015;2:1-14.

6. Chiocca EA, Rabkin SD. Oncolytic viruses and their application to cancer immunotherapy. Cancer Immunology Research 2014;2(4):295-300.

7. Bartlett David L, et al. Oncolytic viruses as therapeutic cancer vaccines. Mol Cancer 2013;12(1):103.

8. Singh PK, Doley J, Kumar GR, Sahoo AP, Tiwari AK. Oncolytic viruses and their specific targeting to tumour cells. Indian J Med Res 2012;136(4):571-584.

9. Ferguson MS, Lemoine NR, Wang Y. Systemic delivery of oncolytic viruses: hopes and hurdles. Adv Virol 2012;2012:1-14.

10. Vacchelli Erika, et al. Trial watch: Oncolytic viruses for cancer therapy. Oncoimmunology 2013;2(6):e24612.

Shankargouda Patil

Associate Professor

Department of Oral Pathology and Microbiology

Faculty of Dental Sciences

MS Ramaiah University of Applied Sciences

Bengaluru, Karnataka, India

Roopa S Rao

Professor and Head

Department of Oral Pathology and Microbiology

Faculty of Dental Sciences

MS Ramaiah University of Applied Sciences

Bengaluru, Karnataka, India

Barnali Majumdar

Postgraduate Student

Department of Oral Pathology and Microbiology

Faculty of Dental Sciences

MS Ramaiah University of Applied Sciences

Bengaluru, Karnataka, India 